
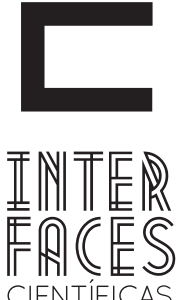

SAÚDE E AMBIENTE

\title{
CONHECIMENTO TEÓRICO-CIENTÍFICO DOS PROFISSIONAIS QUE ATUAM NO CENTRO DE MATERIAL E ESTERILIZAÇÃO NO MUNICÍPIO DE ARACAJU/SERGIPE
}

Jamile e Silva Souza ${ }^{1}$

Emília Cervino Nogueira
Priscila Costa de Sá

\section{RESUMO}

O Centro de Material e Esterilização (CME) é um setor responsável pelo reprocessamento e distribuição de artigos de saúde para as unidades consumidoras. Sem qualidade nos produtos fornecidos pelo CME não seria possível garantir os cuidados adequados à clientela. O enfermeiro tem papel fundamental para o funcionamento do CME, tanto no gerenciamento do setor quanto na seleção e treinamento do pessoal. Objetivo: Identificar o conhecimento teórico-científico dos profissionais que atuam no Centro de Material e Esterilização conforme a Resolução da Diretoria Colegiada (RDC) No 15 de 15 de março de 2012. Métodos: Estudo transversal descritivo de abordagem quantitativa realizado em três instituições ( $\mathrm{Pb}, \mathrm{F}$ e $\mathrm{PV}$ ), por meio de questionário contendo 35 afirmações verdadeiras e falsas acerca da RDC No 15 . Resultados: sessenta e cinco profissionais de enfermagem participaram do estudo, sendo $41,5 \%$ da instituição $\mathrm{Pb}, 30,8 \%$ da $\mathrm{F}$ e $27,7 \%$ da Pv. $63,8 \%$ das respostas estavam certas, $33,8 \%$ estavam erradas e $2,4 \%$ em branco. Os enfermeiros obtiveram acerto de $80 \%$ e os técnicos e auxiliares de enfermagem $62,4 \%$. Conclusão: A maioria dos pesquisados não tinha conhecimento acerca da existência da RDC No 15 e se atualizava através dos seus colegas de trabalho. Verificou-se que os erros mais frequentes coincidiam, por vezes, em duas das três instituições estudadas. Sabendo da responsabilidade que o CME carrega em seus serviços, tem-se também a ciência da importância da atualização para o funcionamento deste.

\section{PALAVRAS-CHAVE}

Esterilização. Papel do Profissional de Enfermagem. Educação Continuada. Normas. 


\section{ABSTRACT}

The Material and Sterilization Center (MSC) is a sector responsible for reprocessing and distribution of health articles for consumer units. Without quality products provided by MSC would not be possible to ensure adequate care for clients. The nurse has a key role in the operation of the MSC, both in industry and in management selection and training of personnel. Objective: To identify the theoretical and scientific professionals who work at the Centre for Material and Sterilization as the Board Resolution (RDC) No. 15 of March 15, 2012. Methods: Cross-sectional study with a quantitative approach carried out in three institutions ( $\mathrm{Pb}, \mathrm{Pr}$ and $\mathrm{F}$ ), through a questionnaire containing 35 true and false statements about the RDC No. 15. Results: sixty-five nurses participated in the study, with $41.5 \%$ of the institution $\mathrm{Pb}, 30.8 \% \mathrm{~F}$ and $27.7 \%$ of $\mathrm{Pv} .63 .8 \%$ of responses were correct, $33.8 \%$ were wrong and $2.4 \%$ blank. Nurses received $80 \%$ hit and technicians and nursing assistants $62.4 \%$. Conclusion: The majority of respondents had no knowledge of the existence of the RDC No. 15 and was updated through their co-workers. It was found that the most frequent errors coincide, sometimes in two of the three institutions studied. Knowing the responsibility that the MSC carries in its services, it has also the importance of science to update its rules.

\section{KEYWORDS}

Sterilization. Role of the Nursing Professional. Continuing Education. Standards.

\section{RESUMEN}

El Centro de Material y Esterilización (CME) es un sector responsable de reprocesamiento y distribución de artículos de salud para las unidades de consumo. Sin productos de calidad proporcionados por el CME no sería posible garantizar una atención adecuada a los clientes. El enfermero tiene un papel clave en el funcionamiento del CME, tanto en la administración del sector como en la selección y entrenamiento del personal. Objetivo: Identificar el conocimiento teórico y científico de las personas que trabajan en el Centro de Material y Esterilización de acuerdo con la Resolución del Consejo (RDC) N ${ }^{\circ} 15$ de 15 de marzo 2012. Métodos: Estudio transversal descriptivo con abordaje cuantitativo, realizado en tres instituciones $(\mathrm{Pb}, \mathrm{Pr}$ y F), utilizando un cuestionario con 35 afirmaciones verdaderas y falsas sobre la RDC N ${ }^{\circ} 15$. Resultados: sesenta y cinco enfermeros participaron del estudio, con el $41,5 \%$ de la institución $\mathrm{Pb}, 30,8 \%$ de la $\mathrm{F}$ y $27,7 \%$ de la Pv. Un 63,8\% de las respuestas estaban correctas, el $33,8 \%$ estaban equivocadas y el $2,4 \%$ estaban en blanco. Los enfermeros alcanzaron un $80 \%$ de respuestas correctas y los técnicos y auxiliares de enfermería un 62,4\%. Conclusión: La mayoría de los encuestados no tenía conocimiento de la existencia de la RDC N ${ }^{\circ} 15$ y se actualizaba a través de sus compañeros de trabajo. Se encontró que los errores más frecuentes coincidían, a veces en dos de las tres instituciones estudiadas. Considerando la responsabilidad que el CME lleva en sus servicios, se nota también la importancia de la actualización de conocimientos para el buen desarrollo de sus actividades.

\section{PALABRAS CLAVE}

Esterilización. El Papel del Profesional de Enfermería . Educación Continua. Normas. 


\section{INTRODUÇÃO}

O Centro de Material e Esterilização (CME) é a unidade funcional destinada ao processamento de produtos para saúde que consiste no conjunto de ações relacionadas à pré-limpeza, recepção, limpeza, secagem, avaliação da integridade e da funcionalidade, preparo, desinfecção ou esterilização, armazenamento e distribuição para as unidades consumidoras (ANVISA, 2012).

A Agência Nacional de Vigilância Sanitária (ANVISA) em sua Resolução da Diretoria Colegiada (RDC) n $n^{0} 307$ de 14 de novembro de 2002, considera o CME como unidade de apoio, que tem como finalidade fornecer artigos adequadamente processados. 0 mesmo deve existir quando houver centro cirúrgico, obstétrico e/ou ambulatorial, hemodinâmica, emergência de alta complexidade e urgência, podendo estar localizado fora do estabelecimento assistencial de saúde. O CME simplificado, só pode existir como apoio técnico a procedimentos que não exijam ambiente cirúrgico para sua realização (ANVISA, 2002).

As atividades executadas pelos profissionais do CME devem ser rigorosamente controladas e organizadas, de modo a evitar qualquer tipo de erro, podendo gerar danos futuros. Segundo Silva e Aguiar (2008), sem qualidade nos produtos fornecidos pelo CME, não seria possível garantir os cuidados adequados à clientela, afinal qualquer falha durante o processo, compromete a esterilização desses materiais, levando riscos de infecção e outras complicações aos pacientes nos períodos trans e pós-cirúrgicos bem como, em procedimentos não cirúrgicos.

O CME necessita para seu adequado funcionamento, tecnologia em equipamentos, postura dos profissionais, e eficiente trabalho em equipe. Alguns fatores relevantes para o comprometimento do trabalho são: infraestrutura, dinâmica das relações humanas, qualificação dos profissionais, pressão inerente ao serviço devido ao processo de trabalho caracterizado pelo modo sequencial das etapas operacionais do reprocessamento de artigos, e necessidade de produtividade (MARTINS et al, 2011; SOBECC, 2009).

Segundo Possari (2010), por se tratar de um dos setores mais importantes do hospital, o CME deve ainda apresentar uma atuação sistematizada, fazendo-se necessário que toda assistência prestada seja embasada em protocolos, rotinas e procedimentos atualizados garantindo a uniformização do atendimento e a organização do setor, como também facilitando o treinamento de funcionários recém-admitidos.

0 enfermeiro tem papel fundamental para o funcionamento do CME. Sua função começa desde o planejamento da unidade até a coordenação, orientação e supervisão das etapas de reprocessamento dos produtos, além de estabelecer conexões com as unidades consumidoras. Outra importante atribuição é a seleção e o treinamento do pessoal, de forma a capacitá- los através da educação continuada, facilitando sua atualização e aquisição dos conhecimentos (SILVA; AGUIAR, 2008).

Em 15 de março de 2012 a ANVISA publicou a Resolução da Diretoria Colegiada n 15 com o objetivo de estabelecer os requisitos de boas práticas para o funcionamento dos serviços que realizam o processamento de produtos para a saúde. Diante disso, buscou-se identificar o conhecimento teórico-científico dos profissionais que atuam no Centro de Material e Esterilização no Município de Aracaju/SE. 


\section{METODOLOGIA}

0 presente estudo teve caráter transversal descritivo, com abordagem quantitativa, tendo sido realizado no Centro de Material e Esterilização de três instituições hospitalares do município de Aracaju/SE, sendo uma filantrópica, denominada "F", uma da rede pública, intitulada " $\mathrm{Pb}$ " e outra da rede privada, chamada "Pv".

A coleta de dados ocorreu no período de outubro a novembro do ano de 2012, nos três turnos (manhã, tarde e noite), tendo início logo após o recebimento do parecer de aprovação do projeto pelo Comitê de Ética em Pesquisa da Universidade Tiradentes, Cadastro no 170912.

A amostra da pesquisa incluiu todos os profissionais de enfermagem que atenderam aos critérios de inclusão, ou seja, ser profissional de Enfermagem de nível médio, técnico ou superior, atuar no setor há mais de seis meses e concordar em participar da pesquisa. Foram excluídos aqueles que se encontravam de folga, férias, atestado médico, licença maternidade, licença prêmio e os que faltaram por ocasião da coleta. Todos os pesquisados assinaram o Termo de Consentimento Livre e Esclarecido (TCLE) concordando em participar do estudo.

\section{RESULTADOS E DISCUSSÃO}

Dos 131 profissionais existentes nas instituições pesquisadas, 65 (49,6\%) participaram da pesquisa, conforme os critérios de inclusão/exclusão, sendo 27 profissionais da instituição "Pb" (41,5\%), 20 da "F" (30,8\%) e 18 da "Pv" (27,7\%).

$\mathrm{Na}$ totalidade dos pesquisados, 62 profissionais são do sexo feminino $(95,4 \%)$ e três do sexo masculino (4,6\%). A prevalência feminina na Enfermagem é
Utilizou-se como instrumento de coleta de dados um questionário elaborado pelas próprias pesquisadoras, que contava com itens para caracterização da amostra como idade, sexo, escolaridade, estado civil, tempo de serviço e cargo Questionava-se, logo após, se o pesquisado possuía conhecimento sobre a existência da RDC $n^{0} 15$ e em seguida, qual o método mais utilizado por eles para se atualizarem. 0 decorrer do questionário expunha 35 afirmações sobre a RDC $\mathrm{n}^{0} 15$, as quais os participantes julgaram ser verdadeiras, marcando um " $V$ ", ou falsas, preenchendo com "F", ou deixaram em branco.

A tabulação dos dados aconteceu com auxílio do programa Statistical Package for the Social Sciences 18 (SPSS), sendo analisados conforme estatística descritiva. As questões foram corrigidas com embasamento científico, fundamentado na RDC $n^{0} 15$. Foi investigado o quantitativo de acertos, erros e questões em branco de cada questionário. Para elaboração dos resultados, trabalhou-se com a frequência absoluta e relativa das variáveis, caracterizando-as de forma geral e individualmente.

uma realidade neste cenário profissional, como ilustra a pesquisa.

Segundo Lopes e Leal (2005), ao retomar aos aspectos sócio-históricos, pode-se dizer que a enfermagem nasce como um serviço organizado pela instituição das ordens sacras. Por longo período, essa marca das ordens religiosas impõe à enfermagem, seu exercício institucional exclusivo e ou majoritariamente 
feminino e caritativo. Apesar dos riscos de eventualidades, mantém-se feminina em todos os níveis, porém não mais exclusivamente.

Em relação ao cargo, 46 dos profissionais entrevistados são auxiliares de enfermagem (70,8\%), 14 são técnicos de enfermagem (21,5\%) e cinco são enfermeiros $(7,7 \%)$. Em todas as instituições prevaleceu 0 cargo de auxiliar de enfermagem.

Foi evidenciado um quantitativo de enfermeiros abaixo do esperado na instituição "Pv", pelo fato de que eles respondem, além do CME, pelo Centro Cirúrgico, e/ou por estarem em um plantão atribulado. Já na instituição "F", a inexistência de enfermeiro está relacionada principalmente à ausência deste no momento da pesquisa, por assumir além do CME, o Centro Cirúrgico e a Unidade Vascular Avançada (UVA), referindo assim uma sobrecarga de trabalho.

No que se refere à idade, foi observado uma predominância na faixa etária de 41 a 50 anos, tanto na instituição "Pb" (51,9\%) quanto na " $\mathrm{F}$ " (50\%). Em contrapartida, a instituição "Pv" apresenta uma prevalência na faixa etária de 21 a 30 anos (55,6\%). Quanto ao quantitativo geral, tem-se como predominante a faixa etária de 41 a 50 anos (38,5\%).

No que diz respeito ao tempo de serviço, 50,8\% estão dentro de um intervalo de 6 meses a 5 anos quando analisado o geral das três instituições. Estudadas isoladamente é visto que na instituição "Pb" a maior parte dos profissionais atuam no CME no intervalo de 6 a 10 anos (33,3\%), enquanto nas instituições "F" e "Pv" encontram-se trabalhadores, em maior número, com tempo de serviço que varia de 6 meses a 5 anos, tendo uma frequência relativa de $50 \%$ e $83,3 \%$, respectivamente.

Estes dados são traduzidos de formas divergentes no que tange a sua justificativa, estando a prevalência da instituição "F" ligada à presença de funcionários problemáticos ou com alguma deficiência, o que aumenta a rotatividade de profissionais no setor, e a da "Pv" relacionada a busca por profissionais capacitados e qualificados para a atuação no CME, visto que todos os trabalhadores desta passam por um período de treinamento.

Bartolomei e Lacerda (2006), afirmam que até os anos 70, o CME não era valorizado, sendo localizado em ambientes inadequados e com recursos insuficientes, do mesmo modo era o trabalho da enfermagem nesse setor. Esta fala é complementada pela alocução de Machado e Gelbcke (2009), os quais dizem que outra razão para a desvalorização do CME é o remanejamento de profissionais que não se adaptaram à assistência direta ao cliente, por problemas de relacionamento interpessoal, assiduidade ou deficiência de conhecimento.

Quando questionado se os participantes tinham conhecimento sobre a existência da RDC n015, verificou-se que 45 profissionais $(69,2 \%)$ dos 65 estudados não conheciam a referida RDC. É visto que $100 \%$ dos profissionais estudados da instituição " $F$ " desconhecem a existência da RDC no 15 (Figura 1), fato que induz o questionamento acerca da qualidade dos artigos processados e distribuídos pelo CME naquela instituição.

Observou-se que a maioria dos profissionais da instituição " $\mathrm{Pb}$ " $(66,7 \%)$ também não tinha conhecimento da existência da resolução citada. Já na "Pv" dos 18 profissionais estudados, $11(61,1 \%)$ sabiam da existência da RDC $n^{0} 15$ (Figura 1), levando-se em conta a ocorrência de uma educação em serviço sobre esta resolução na semana que antecedeu a visita das pesquisadoras, segundo informado por funcionários do setor. 
Figura 1 - Comparação do conhecimento acerca da existência da RDC No 15 entre as instituições Pb (Pública), F (Filantrópica) e Pv (Privada).

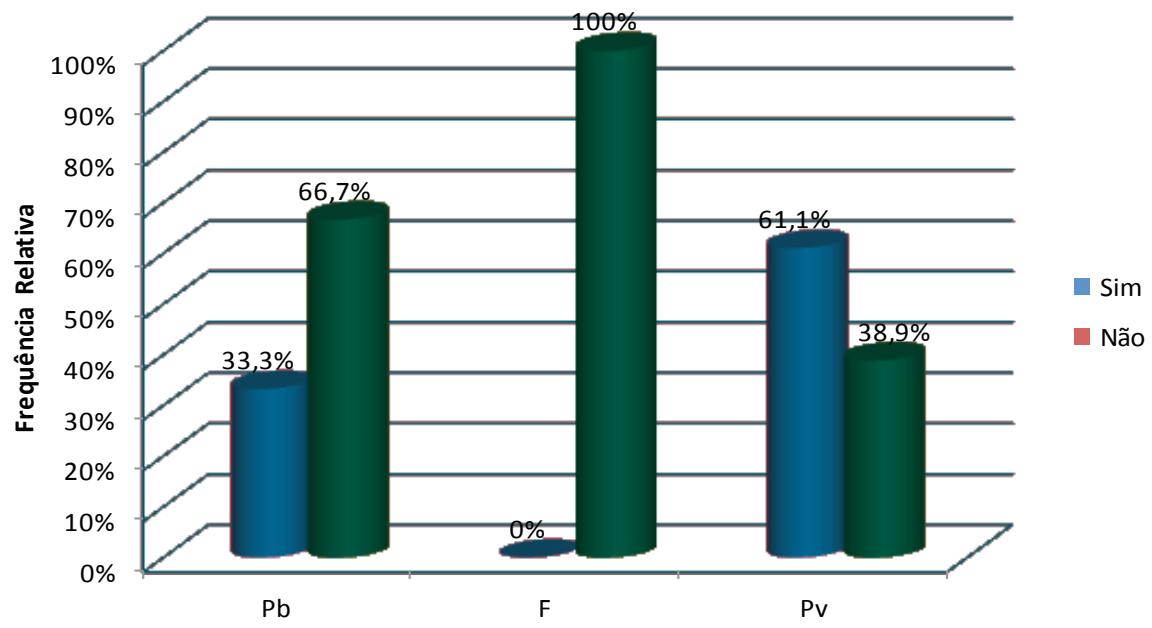

Fonte: Dados da Pesquisa, 2012.

Silva e Aguiar (2008, p.13) fundamentam o questionamento acima quando afirmam que "A qualidade da assistência prestada [...] tem relação direta com os produtos fornecidos pela CME, sem os quais não seria possível garantir os cuidados adequados à clientela". A necessidade de treinamento e desenvolvimento dos profissionais do CME tem sido corroborada pelas mudanças ocorridas ao longo da evolução do setor, por este ser de alta complexidade, a incorporação de mão de obra desqualificada pode resultar em descrédito nas ações de enfermagem, além de agravos ao cliente, acrescenta Possari (2010).

Percebeu-se, mediante análise do método mais utilizado pelos profissionais para manterem-se atualizados, assunto tratado mais a frente, que nas instituições "Pb" e "F", a possível causa do desconhecimento por parte destes seria a falta de capacitações, treinamentos e atualizações tanto iniciais quanto periódicas, enquanto que na "Pv" a prática da educação em serviço, ofertada pelo enfermeiro, é regular, o que explica o conhecimento que os profissionais da instituição detêm acerca da RDC comentada.
Quanto à análise do conhecimento da existência da RDC no 15 pelas categorias, verificou-se que um (20\%), dos cinco enfermeiros participantes, desconhecia a existência da referida RDC, fato que chama a atenção levando-se em conta a importância da atualização na prática das funções deste profissional, e considerando-se a afirmação de Silva e Aguiar (2008, p.25), a qual diz que "[...] os procedimentos de enfermagem na assistência direta, que são considerados visíveis, dependem do invisível, mas que é essencial: o trabalho do enfermeiro em CME [...]".

Stekel (2011) traz em sua dissertação de mestrado, uma consideração relevante para este estudo. Em sua pesquisa, a maioria $(72,4 \%)$ dos auxiliares de enfermagem possuía curso técnico. Acordando com os dados de Stekel, resolveu-se analisar e apresentar os resultados fazendo a junção dessas duas categorias. Os técnicos e auxiliares de enfermagem demonstraram não deter conhecimento sobre a existência da RDC no 15 , apresentando uma frequência relativa igual a $73,3 \%$. 
A maioria dos profissionais estudados se atualizava por meio dos colegas de trabalho $(29,8 \%)$, seguindo, as instituições "Pb" e "F", a mesma via de regra, expondo uma porcentagem de $34,1 \%$ e $52,4 \%$, respectivamente. A única que, como vem ocorrendo, apresenta discrepância nos resultados, quando comparada as demais, é a "Pv", onde o método mais comum é a educação em serviço (40,5\%).

Compartilhando da afirmação de Silva, Conceição e Leite (2008, p.15), dizem que "a educação continuada é o componente essencial dos programas de formação e desenvolvimento de recursos humanos das instituições" atenta-se para o fato desta só ser aplicada de forma rigorosa na instituição "Pv", decorrendo grande parte desta rigorosidade, da exigência, por parte dos clientes, por um serviço de qualidade e que não lhes ofereça riscos.

Ao se tratar das 35 questões de verdadeiro ou falso, aplicadas com a amostra de 65 profissionais da área de enfermagem, atuando no CME há mais de 6 meses, obteve-se um total de 2275 respostas, onde
1451 estavam certas (63,8\%), 769 erradas (33,8\%) e 55 em branco $(2,4 \%)$.

A instituição com o maior índice de questões certas foi a "Pv", sendo que de 630 respostas, 438 (69,5\%) estavam corretas (Figura 2). Conforme Silva e Aguiar (2008) cabe ao enfermeiro capacitar os funcionários da sua equipe, que estejam sob sua responsabilidade por meio da educação continuada, servindo ele, como facilitador da aquisição de saber e atualização. Dessa forma, compreende-se que, no contexto geral, o bom resultado apresentado nestes questionários, advém das capacitações exigidas pela instituição e realizadas periodicamente pelo enfermeiro.

Já a "F" se destacou como a instituição com o maior número de erros no questionário, sendo que dentre 700 respostas, 275 estavam erradas (39,3\%) (Figura 2), apontando uma preocupação nos serviços ofertados pelo CME. Este fato pode estar relacionado à falta de treinamento desses profissionais para integração no setor, além da ausência de um enfermeiro fixo para capacitação e supervisão de suas atividades.

Figura 2 - Comparação do total de respostas certas, erradas e em branco entre as instituições Pb (Pública), F (Filantrópica) e Pv (Privada)

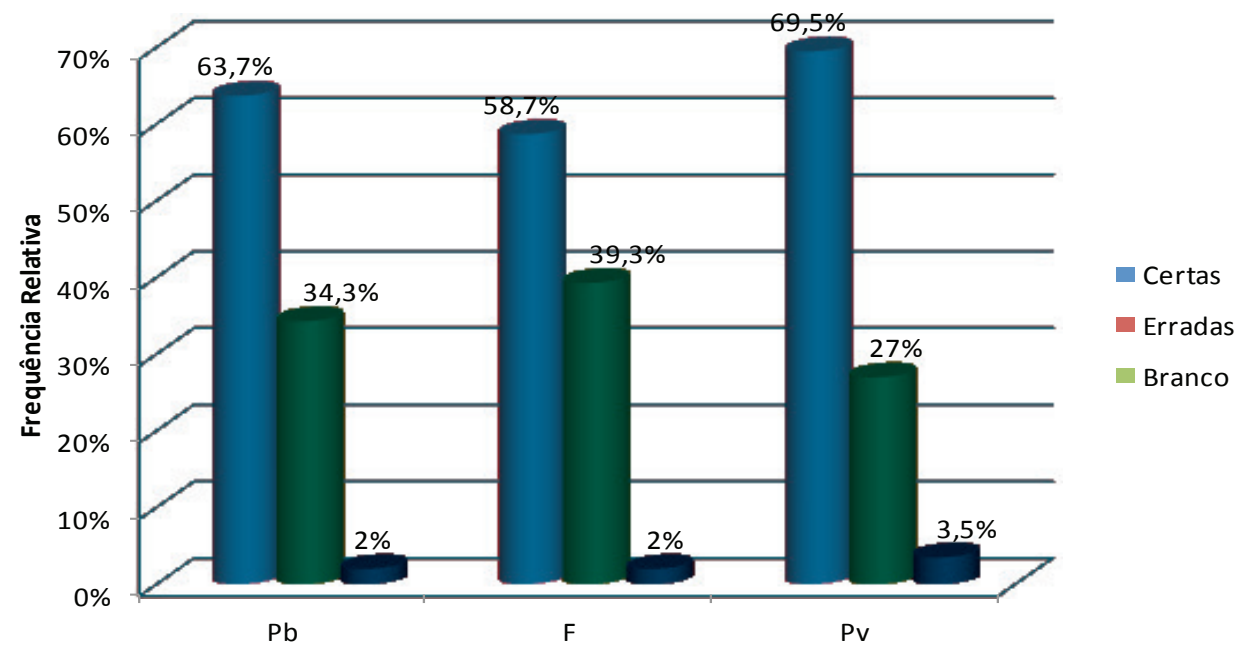

Fonte: Dados da Pesquisa, 2012. 
No que se refere às respostas por categoria profissional, participaram da pesquisa cinco enfermeiros $(7,7 \%)$, o que representa 175 respostas. Destas, acertaram 140 (80\%), com 34 erros (19,4\%) e uma questão em branco (0,6\%) (Figura 3). Na instituição "Pv", dois enfermeiros responderam ao questionário, onde em um total de 70 respostas, $60(85,7 \%)$ estavam corretas.

Os técnicos ou auxiliares de enfermagem representaram 92,3\% da amostra, totalizando 2100 res- postas. Destas, 1311 estavam certas (62,4\%), 735 estavam erradas (35\%) e 54 em branco (2,6\%) (Figura $3)$. É compreensível que somente profissionais qualificados e com capacitações permanentes possam provocar mudanças significativas na organização do trabalho em saúde e no CME, mais particularmente no controle de infecção hospitalar e na qualidade da assistência ao cliente (MACHADO; GELBCKE, 2009).

Figura 3 - Comparação do total de respostas certas, erradas e em branco entre as categorias profissionais

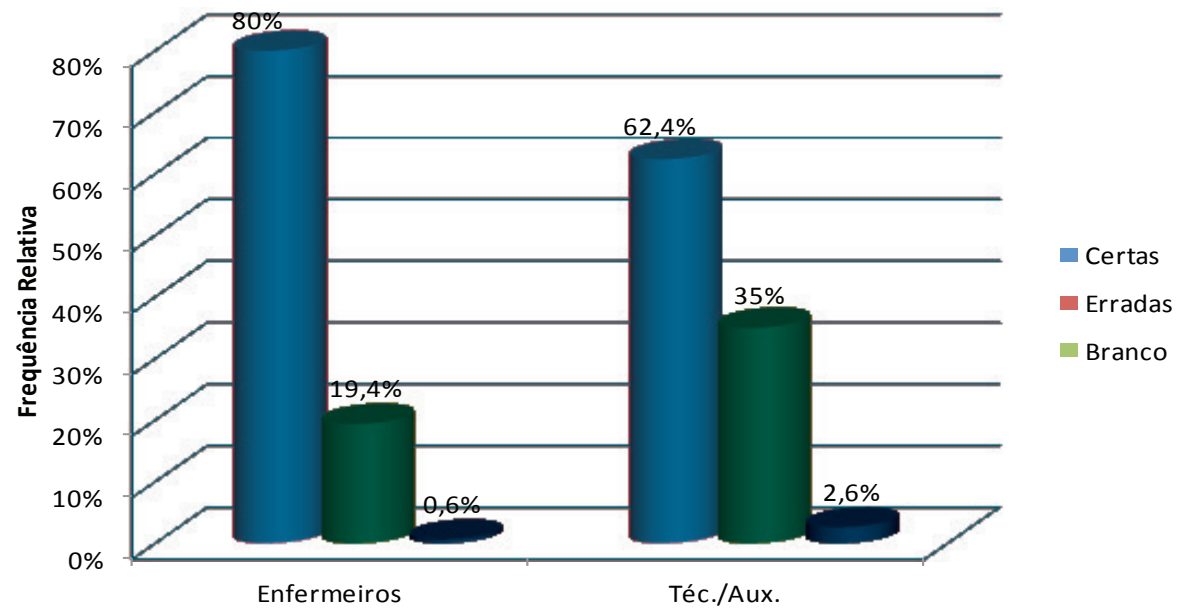

Fonte: Dados da Pesquisa, 2012.

É de fundamental importância, que os profissionais designados para atuar no CME, possuam capacidades interpessoais e técnicas, objetivando um processo de trabalho ativo e valorização das potencialidades da equipe, de modo a contribuir a segurança dos materiais esterilizados (AGUIAR; SOARES; SILVA, 2009).
Ao analisar a taxa de erros dos profissionais por instituição, foram consideradas as respostas em branco como erradas, pois essa dúvida por parte do entrevistado leva a pensar que ele não soube responder à questão. A Figura 4 apresenta as questões em que mais de $50 \%$ das respostas não estavam corretas. 


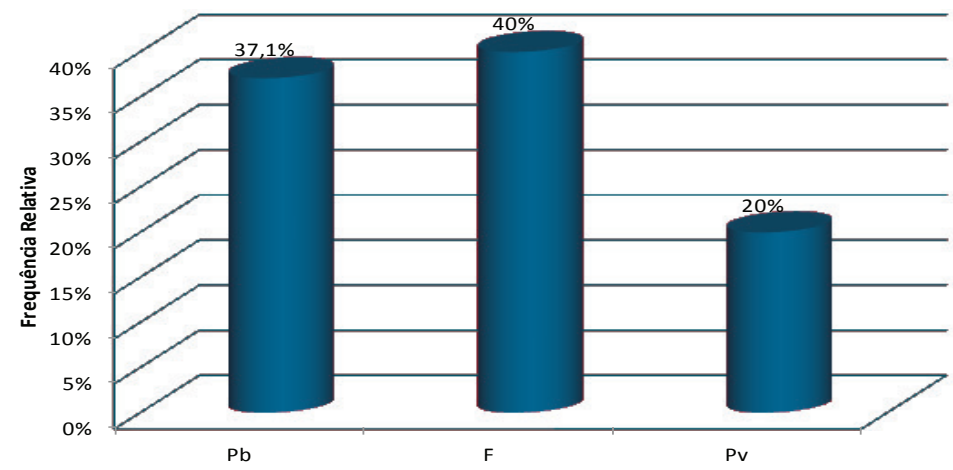

Fonte: Dados da Pesquisa, 2012.

$\mathrm{Na}$ instituição "Pb", verificou-se que 13 (37,1\%) das 35 questões, apresentaram esse percentual. Nesta mesma instituição, 25 (92,6\%) dos 27 profissionais entrevistados, erraram a terceira questão, concordando que a data limite de uso do produto esterilizado compreende um prazo estabelecido pela ANVISA.

Segundo RDC $n^{0} 15$ (2012), essa data limite deverá ser estabelecida em cada instituição, baseado em um plano de avaliação da integridade das embalagens e fundamentado na sua resistência, eventos relacionados ao seu manuseio (estocagem em gavetas, empilhamento de pacotes, dobras das embalagens), condições de umidade e temperatura, segurança da selagem e rotatividade do estoque armazenado.

O mesmo ocorreu com a oitava questão, onde eles afirmaram que o CME deve dispor de um sistema de informação manual ou automatizado com registro do monitoramento e controle das etapas de processamento, devendo esses registros ser arquivados, de forma a garantir a sua rastreabilidade por até um ano para efeitos de inspeção sanitária. A RDC n 15 (2012) impõe que o CME e a empresa processadora devem dispor sim desse sistema, porém esses registros devem ser arquivados, por um prazo mínimo de "CINCO" anos, para efeitos de inspeção sanitária.

No que diz respeito à instituição "F", 14 questões (40\%) apresentaram mais de $50 \%$ de erro, dentre estas a mais requente foi a mesma exposta pela instituição "Pb", ou seja, a terceira questão, onde todos os profissionais estudados (100\%) não acertaram a resposta.

A instituição "Pv" foi a que apresentou o menor percentual, no que tange às questões com mais de $50 \%$ de erros, onde sete (20\%) das 35 questões apresentaram esse valor. Assim como nas outras duas instituições, a terceira questão foi a que possuiu o maior índice de erro. Acredita-se que essa porcentagem de erro nas três instituições, provém da confusão por parte dos participantes no que se relaciona ao órgão responsável pela definição do prazo da data limite de uso do produto esterilizado. 


\section{CONCLUSÃO}

A maioria dos pesquisados não possuía conhecimento acerca da RDC $n^{0} 15$ e a atualização por parte dos mesmos era realizada, em maior número, através dos colegas de trabalho.

Quando observado separadamente o conhecimento sobre a existência da referida RDC, percebeu-se que a única instituição onde a maioria de seus profissionais tinha conhecimento foi a instituição "Pv", e relacionado às categorias, os enfermeiros, em sua grande maioria, sabiam da existência da RDC no 15, enquanto que grande parte dos técnicos e auxiliares desconheciam. Porém, chama a atenção o fato de um enfermeiro não ter conhecimento, sequer, da existência da RDC comentada, direcionando o pensamento a questionar o tipo de reprocessamento que está sendo realizado.

A instituição "Pv" exibe como método de atualização predominante a Educação em Serviço, que, após o exposto neste estudo e com base em estudos anteriores, pode-se afirmar ser um instrumento fundamental para o adequado funcionamento do CME. A inexistência da prática deste método nas instituições "Pb" e "F", sendo mais comum a atualização por meio dos colegas de trabalho, pode ser considerada a cau- sa do desconhecimento sobre a RDC no 15 por parte dos profissionais.

Verificou-se que os erros mais frequentes relacionavam-se ao órgão responsável pela definição do prazo da data limite de uso do produto esterilizado, e ao tempo necessário que os registros de monitoramento devem ficar arquivados para efeito de inspeção sanitária. Acredita-se que o fato advém da confusão feita pelos profissionais de enfermagem estudados acerca dos temas citados acima.

Sabendo da responsabilidade que o CME carrega em seus serviços, tem-se também a ciência da importância da atualização para o funcionamento deste. Desta forma, baseando-se nos dados obtidos, conclui-se que uma grande parte dos profissionais estudados não se encontrava atualizada e consequentemente capacitada para atuar no setor em foco. Sendo assim, resta um alerta e questiona-se que tipo de assistência está sendo prestada à clientela, buscando, este estudo, conscientizar os profissionais de enfermagem da importância da atuação de trabalhadores qualificados e capacitados, e ressaltar a extrema relevância da Educação Permanente destes profissionais.

\section{REFERÊNCIAS}

AGUIAR, B. G. C.; SOARES, E.; SILVA A. C. Evolução das Centrais de Material e Esterilização: História, Atualidades e Perspectivas Para a Enfermagem. Reflexiones - Ensayos, n. 15, p. 1-6, 2009.

BRASIL. ANVISA. Agência Nacional de Vigilância Sanitária. Resolução da Diretoria Colegiada No 15 de 15 de março de 2012. Disponível em: <http://www.brasilsus.com.br/legislacoes/gm/112548-15.html> Acesso em: 17 maio 2012.

BRASIL. Resolução da Diretoria Colegiada no 307 de 14 de novembro de 2002. Disponível em: < http://www. anvisa.gov.br/legis/resol/2002/307_02rdc.htm> Acesso em: 21 maio 2012. 
BARTOLOMEI, S. R. T.; LACERDA, R. A. 0 enfermeiro da Central de Material e Esterilização e a percepção do seu papel social. Rev Gaúcha Enferm. Porto Alegre (RS) 2006 jun; 27(2):258-65.

LOPES, M. J. M.; LEAL, S. M. C. A feminização persistente na qualificação profissional da enfermagem brasileira. Cadernos Pagu (24), janeiro-junho de 2005, pp.105-125.

MACHADO, R. R.; GELBCKE, F. L. Que brumas impedem a visibilização do Centro de Material e Esterilização? Texto Contexto Enfermagem. Florianópolis, 2009 Abr-Jun; 18(2): 347-54.

MARTINS, V. M. F. et al. Forças impulsoras e restritivas para trabalho em equipe em um Centro de Material e Esterilização de hospital escola. Revista Escola Enfermagem USP. 2011; 45(5):1183-90.

POSSARI, J. F. Centro de Material e Esterilização: planejamento, organização e gestão. 4. ed. rev. atual. ampl. São Paulo: látria, 2010.166 p.

SILVA, A. C.; AGUIAR, B. G. C. O Enfermeiro na Central de Material e Esterilização: uma visão das unidades consumidoras. Revista Enfermagem UERJ. Rio de Janeiro, 2008 jul/set; 16(3):377-81.

SILVA, M. F.; CONCEIÇÃO, F. A.; LEITE, M. M. J. Educação Continuada: um levantamento de necessidades da equipe de enfermagem. 0 Mundo da Saúde. São Paulo: 2008: jan/mar 32(1):47-55.

STEKEL, L. M. C. Estresse e coping entre auxiliares e técnicos de enfermagem de um hospital universitário. 2011. 99 f. Dissertação (Mestrado em Enfermagem) - Programa de Pós-Graduação em Enfermagem, Centro de Ciências da Saúde, Universidade Federal de Santa Maria, Rio Grande do Sul.

SOBECC. Sociedade Brasileira de Enfermeiros de Centro Cirúrgico, Recuperação Anestésica e Centro de Material e Esterilização. Práticas Recomendadas SOBECC. 5 ed. São Paulo, 2009. 304 p.

Recebido em: 18 de fevereiro de 2013 Avaliado em: 12 de abril de 2013 Aceito em: 26 de abril de 2013
1 Enfermeira pela Universidade Tiradentes - UNIT. Aracaju, Sergipe, Brasil. E-mail: jamilesouza_enfa@yahoo.com Tel: (79)9998-9411 / (79)91236599.

2 Enfermeira pela Universidade Tiradentes - UNIT. Aracaju, Sergipe, Brasil. E-mail: priscilasa_enfa@yahoo.com Tel: (79)9831-5377 / (79)91257173.

3 Enfermeira. Mestre em Ciências da Saúde. Docente da Universidade Tiradentes - UNIT. Aracaju, Sergipe, Brasil. Orientadora do Presente Artigo. E-mail: ecervino.n@ig.com.br Tel: (79)9987-4642. 